\title{
On the Relations between Gravity and BF Theories ${ }^{\star}$
}

\author{
Laurent FREIDEL ${ }^{\dagger}$ and Simone SPEZIALE ${ }^{\ddagger}$ \\ $\dagger$ Perimeter Institute, 31 Caroline St N, Waterloo ON, N2L 2Y5, Canada \\ E-mail: lfreidel@perimeterinstitute.ca \\ ‡ Centre de Physique Théorique, CNRS-UMR 7332, Luminy Case 907, 13288 Marseille, France \\ E-mail: simone.speziale@cpt.univ-mrs.fr
}

Received January 23, 2012, in final form May 18, 2012; Published online May 26, 2012

http://dx.doi.org/10.3842/SIGMA.2012.032

\begin{abstract}
We review, in the light of recent developments, the existing relations between gravity and topological BF theories at the classical level. We include the Plebanski action in both self-dual and non-chiral formulations, their generalizations, and the MacDowellMansouri action.

Key words: Plebanski action; MacDowell-Mansouri action; BF gravity; TQFT; modified theories of gravity

2010 Mathematics Subject Classification: 83C45
\end{abstract}

\section{Introduction}

It is an intriguing fact that general relativity can be given a polynomial action principle which relates it to topological BF theory. The fundamental variable is a connection, and the metric is a derived quantity. For BF theory alone, a Lagrange multiplier, $B$, enforces the curvature of the connection to be constant. This type of theories require no metric to be formulated, and posses no local degrees of freedom. The relation with general relativity is established through the extraction of metric degrees of freedom from the fundamental fields. There are mainly two ways of achieving this that have appeared in the literature, based on original work by Plebanski [77] and by MacDowell and Mansouri [66]. The two ways differ in the choice of gauge group, and in the way the metric is recovered - from either the $B$ field or the connection. In this review, we will go through the basic aspects of these two approaches, as well as some recent developments. We restrict our attention to four spacetime dimensions. For the connection between BF theory and gravity in three dimensions, see [22, 30, 98], and [47] in dimensions higher than four.

\section{BF theory}

Let us begin with some general aspects of BF theory [22]. We consider a principal bundle over the spacetime manifold $M$, with local group a Lie group $G$ and connection $\omega$, with curvature $F(\omega)$, and the following action,

$$
S(B, \omega)=\int \operatorname{tr} B \wedge F-\frac{\lambda}{2} \operatorname{tr} B \wedge B
$$

where $B$ is a 2 -form in the adjoint representation of $G$ and $\operatorname{tr}$ denotes the scalar product in the algebra. $\lambda$ is a dimensionless constant. Notice that the action is well-defined without any reference to a metric structure on $M$. The field equations are

$$
F(\omega)=\lambda B, \quad d_{\omega} B=0 .
$$

\footnotetext{
*This paper is a contribution to the Special Issue "Loop Quantum Gravity and Cosmology". The full collection is available at http://www.emis.de/journals/SIGMA/LQGC.html
} 
The action is invariant under local gauge transformations,

$$
\delta_{\alpha}^{G} B=[B, \alpha], \quad \delta_{\alpha}^{G} \omega=d_{\omega} \alpha
$$

and under the following shift symmetry,

$$
\delta_{\eta}^{S} B=d_{\omega} \eta, \quad \delta_{\eta}^{S} \omega=\lambda \eta
$$

The total symmetry group is thus the semi-direct product of these two groups, and it is the same for all values of $\Lambda$. Overall, there are $N$ gauge parameters in the algebra-valued scalars $\alpha^{i}$, and $4 N$ in the algebra-valued 1 -forms $\eta_{\mu}^{i}$. The system has so much symmetry that all solutions are locally gauge equivalent, and local degrees of freedom are absent. This is most directly established in the canonical formalism.

The phase space of (1) is spanned by the $6 N$ variables $\left(\omega_{a}^{i}, \Pi_{i}^{a}:=\frac{1}{2} \epsilon^{a b c} B_{i b c}\right)$, where $a=1,2,3$ are space indices. The action takes the form

$$
S=\int \Pi_{i}^{a} \dot{\omega}_{a}^{i}+\omega_{0}^{i} \mathcal{G}_{i}+B_{0 a}^{i} \mathcal{C}_{i}^{a}
$$

and the (fully constrained) dynamics is given by the $N$ Gauss constraints $\mathcal{G}_{i}$ and $3 N$ "Hamiltonian" constraints $\mathcal{C}_{i}^{a}$,

$$
\mathcal{G}_{i}=D_{a} \Pi_{i}^{a}=0, \quad \mathcal{C}_{i}^{a}=\frac{1}{2} \epsilon^{a b c} F_{i b c}(\omega)-\lambda \Pi_{i}^{a}=0 .
$$

As a consequence of the Bianchi identity, $D_{a} \mathcal{C}_{i}^{a} \equiv 0$, thus there are only $3 N$ independent constraints. The algebra of constraints is

$$
\left\{C(N), C\left(N^{\prime}\right)\right\}=0, \quad\left\{G(\alpha), G\left(\alpha^{\prime}\right)\right\}=G\left(\left[\alpha, \alpha^{\prime}\right]\right), \quad\{C(N), G(\alpha)\}=C([N, \alpha]),
$$

where $C(N)=\int N_{a}^{i} \mathcal{C}_{i}^{a}, G(\alpha)=\int \alpha^{i} \mathcal{G}_{i}$. We see that they form a first class system, thus they reduce the phase space by $2 \times 3 N=6 N$ dimensions, leaving a zero-dimensional space of solutions. Hamiltonian systems with linearly dependent constraints are called reducible [22].

The symmetry group also includes the action of diffeomorphisms, which manifestly leaves (1) invariant. Diffeomorphisms are expressed as special combinations of gauge and shift transformations $[26,48]$. Recalling that the Lie derivative of a 1 -form verifies $\mathcal{L}_{\xi} \omega=i_{\xi} d \omega+d\left(i_{\xi} \omega\right)$, where $i_{\xi}$ denotes the inner product with the vector $\xi$, it is easily checked that

$$
\delta_{\xi}^{D} B=d_{i_{\xi} \omega}^{G} B+\delta_{i_{\xi} B}^{S} B+i_{\xi}\left(d_{\omega} B\right), \quad \delta_{\xi}^{D} \omega=d_{i_{\xi} \omega}^{G} \omega+\delta_{i_{\xi} B}^{S} \omega+i_{\xi} F .
$$

Accordingly, the canonical generators of hypersurface deformations can be expressed as linear combinations of $(3)$.

In spite of the lack of local dynamics, BF theory has many interesting applications. The subject of this review is the classical relation to general relativity, but the action has been used also in connection with Yang-Mills theory (e.g. [31]). Concerning the quantum theory, we refer the reader to [22] for the continuum path integral quantization and its relation to the measure of flat connections and Reidemeister torsion, and to [10, 13, 25, 75, 99] for the discrete path integral and its relation to spin foam models for quantum gravity.

\section{Self-dual Plebanski action}

A way to relate BF theory to general relativity is to work with the local gauge group $\mathrm{SU}(2)$, seen as a chiral subgroup of the Lorentz group. The fundamental fields are $\left(B^{i}, \omega^{i}\right)$, with 
$i=1,2,3$ indices in the adjoint representation. Then one can write a densitized symmetric tensor as $[28,94]$

$$
\sqrt{|g|} g_{\mu \nu}=\frac{1}{12} \epsilon_{i j k} \epsilon^{\alpha \beta \gamma \delta} B_{\mu \alpha}^{i} B_{\beta \gamma}^{j} B_{\delta \nu}^{k} .
$$

A theorem by Urbantke states that if the bilinear density $m^{i j} \equiv B^{i} \wedge B^{j}$ is invertible as a matrix in the $i j$ indices, then also the Urbantke metric (4) is invertible, and furthermore $B$ is self-dual (for $\operatorname{det} m>0$, else antiself-dual) with respect to it, that is $B_{\mu \nu}^{i}= \pm 1 /(2 \sqrt{|g|}) \epsilon_{\mu \nu}{ }^{\rho \sigma} B_{\rho \sigma}^{i}$. See e.g. [45] for a recent proof. Therefore, provided the $B$ field is not degenerate in the above sense, we have a natural way to introduce an invertible metric as a composite object. For $B$ real, compatible with the case of Euclidean signature, one automatically obtains a real metric with positive determinant ${ }^{1}$. For $B$ complex, as required by the chiral splitting of the Lorentz group with Lorentzian signature, one obtains a complex metric with negative determinant, and additional reality conditions need to be given [28].

To deal with this formalism, it is useful to introduce a tetrad $e^{I}$ for the metric (4),

$$
g_{\mu \nu}=e_{\mu}^{I} e_{\nu}^{J} \eta_{I J}
$$

and define the Plebanski 2-forms $\Sigma_{\epsilon}^{i} \equiv P_{\epsilon I}^{i} e^{I} \wedge e^{J}=e^{0} \wedge e^{i}+\sqrt{\sigma} \epsilon / 2 \epsilon_{j k}^{i} e^{j} \wedge e^{k}$, where $\sigma= \pm$ is the spacetime signature, $\epsilon= \pm$ and $P_{( \pm) I J}^{a}$ are the projectors on the left- and right-handed $\mathfrak{s u}(2)$ subalgebras, according to the isomorphism $\mathfrak{s o}(3, \sigma) \cong \mathfrak{s u}(2) \oplus \mathfrak{s u}(2)$, and we have fixed the time gauge in the internal indices. These forms have the property that the left-handed part (resp. right-handed) is simultaneously self-dual (resp. antiself-dual) in the spacetime indices with respect to $e$. The set $\Sigma_{\epsilon}^{a}$ provides an orthogonal basis for the 6-dimensional space of 2 -forms. Combining this fact with Urbantke's theorem, we conclude that a generic, albeit non-degenerate, $B$ field can be written as a linear combination of Plebanski's 2-forms. This can be conveniently parametrized as follows,

$$
B_{( \pm)}^{i}=\eta b_{a}^{i} \Sigma_{( \pm)}^{a}, \quad \operatorname{det} b_{a}^{i}=1, \quad \eta= \pm,
$$

where the unimodularity of $b_{a}^{i}$ can always be we assumed, as we did here, thanks to the conformal invariance of the notion of self-duality. The parametrization (6) helps to appreciate the relation between $\mathrm{SU}(2) \mathrm{BF}$ theory and general relativity: it shows how the $B$ field can equivalently be parametrized in terms of a metric $g_{\mu \nu}$ together with the eight components of the unimodular "internal triad" $b_{a}^{i}$, and a sign. From the "triad" $b_{a}^{i}$, one can define the unimodular "internal metric" $q_{a b}:=b_{a}^{i} b_{b}^{j} \delta_{i j}$, given by an SL(3)-rotated form of the identity. Comparing (4) with (5), we see that the $B$ field plays the role of a chiral "cubic root" of the metric, with the SL(3) group formed by the $b_{a}^{i}$ replacing the internal Lorentz group in (5) $[90]^{2}$.

The relation with general relativity can now be easily established: It suffices to impose the condition that $b_{a}^{i} \in \mathrm{SO}(3)$, or equivalently that $q_{a b}=\delta_{a b}$, and the BF action (1) immediately reduces to ( $\eta$ times) the Einstein-Cartan action in self-dual variables, with cosmological constant $\Lambda=-3 \eta \lambda$. The required condition on $b$ can equivalently be written as

$$
B^{i} \wedge B^{j}=\frac{1}{3} \delta^{i j} B_{k} \wedge B^{k}
$$

a form known as Plebanski or metricity constraints. Using a traceless symmetric scalar $\varphi_{i j}$ as a Lagrange multiplier, the constraints can be included in the action,

$$
S(B, \omega)=\int B_{i} \wedge F^{i}(\omega)-\frac{\lambda}{2} B_{i} \wedge B^{i}+\frac{1}{2} \varphi_{i j} B^{i} \wedge B^{j} .
$$

\footnotetext{
${ }^{1}$ Notice that this includes the case of Kleinian signature $(++--)$. The latter and the Euclidean case are distinguished by the signature of $m^{i j}$, which is respectively Lorentzian and Euclidean [45].

${ }^{2}$ This picture can be further extended to the whole GL(3) group [20].
} 
This is Plebanski's self-dual formulation of general relativity [28, 79], to which it is equivalent in the sector of non-degenerate $B$ fields $^{3}$. The role of the constraints is to freeze the scalar degrees of freedom $b_{a}^{i}$, and leave only the Urbantke metric as a dynamical field. In the case of Lorentzian signature, one takes complex $B$ fields, and adds reality conditions on the metric [28].

It is also instructive to see the equivalence at the level of the field equations. To that end, let us fix the solution $B=\eta \Sigma_{(+)}(e)$ to the constraints (the equivalence in the other sectors is analogous to what follows). The compatibility condition $d_{\omega} B=0$ is then solved by the (lefthanded part of the) spin connection $\omega(e)$, and its curvature yields the left-handed part of the Riemann tensor, $F_{\mu \nu}^{i}(\omega(e))=P_{(+)}^{i} \rho \sigma R^{\rho \sigma}{ }_{\mu \nu}(e)$. As such, it can be decomposed into its tracefree Weyl part, and its Ricci part, by projecting also the second pair of indices, $F_{\mu \nu}^{i}(\omega(e))=$ $f_{a}^{i} P_{(+)}^{a} \mu \nu+\bar{f}_{a}^{i} P_{(-)}^{a} \mu \nu$. The symmetric matrix $f^{i}{ }_{a}$ contains the five Weyl components plus the trace of Ricci, and the generic matrix $\bar{f}_{a}^{i}$ contains the nine components of the trace-free Ricci tensor. We now look at the remaining field equations,

$$
F^{i}=\lambda B^{i}-\varphi_{j}^{i} B^{j}
$$

Using the above decomposition of $F$ and the self-duality of $B$, these equations are equivalent to $\bar{f}_{a}^{i}=0, \operatorname{tr} f_{a}^{i}=3 \eta \lambda$, with the remaining components of $f^{i}{ }_{a}$ free. These are precisely the Einstein's equations, namely $R_{\mu \nu}-\frac{1}{4} g_{\mu \nu} R=0, R=-4 \Lambda$, Weyl free, with $\Lambda=-3 \eta \lambda$.

The use of self-dual variables has been a fertile ground for a number of applications in general relativity, especially in the study of exact solutions and integrability, see e.g. [40].

Canonical analysis. The canonical analysis of the theory [28] is similar to the BF case treated earlier. The canonical variables are the same pair $\left(\omega_{a}^{i}, \Pi_{i}^{a}:=\frac{1}{2} \epsilon^{a b c} B_{i b c}\right)$, the system is fully constrained, and the constraints now read

$$
\begin{aligned}
& \mathcal{G}_{i}=D_{a} \Pi_{i}^{a}=0, \quad \mathcal{C}_{i}^{a}=\frac{1}{2} \epsilon^{a b c} F_{i b c}(\omega)-\lambda \Pi_{i}^{a}+\varphi_{i j} \Pi^{a j}=0, \\
& \mathcal{S}^{i j}=\Pi^{a(i} B_{0 a}^{j)}-\frac{1}{3} \delta^{i j} \Pi_{k}^{a} B_{0 a}^{k}=0 .
\end{aligned}
$$

The main novelty is the fact that together with the Hamiltonian, the metricity constraints $\mathcal{S}$ form a second class system, that is, their Poisson brackets do not vanish on the constraint surface. In this situation, one typically proceeds solving explicitly the part of the constraints which is second class, and derive a reduced action in terms of only first class constraints. Assuming that the density-weight $1 \Pi_{i}^{a}$ has an inverse, $\Pi_{i}^{a} \Pi_{b}^{i}=\delta_{b}^{a}$, we can solve them by fixing the value of the Lagrange multiplier $B_{0 a}^{i}$

$$
B_{0 a}^{i}=\sqrt{\operatorname{det} \Pi}\left(\delta^{i j} N+\epsilon^{i j k} N_{k}\right) \Pi_{a j} .
$$

Plugging the result back into the action, one finds

$$
S=\int \Pi_{i}^{a} \dot{\omega}_{a}^{i}+\omega_{0}^{i} \mathcal{G}_{i}+N \mathcal{H}+N^{a} \mathcal{H}_{a}
$$

where $\mathcal{G}$ is the same Gauss constraint as before, $\mathcal{H}_{a}=\Pi_{i}^{b} F_{a b}^{i}$ is the vector constraint, and

$$
\mathcal{H}=\frac{1}{\sqrt{\operatorname{det} \Pi}} \epsilon_{i j k} F_{a b}^{i} \Pi^{a j} \Pi^{b k}-\lambda \sqrt{\operatorname{det} \Pi}
$$

is the scalar Hamiltonian constraint. The result is the Ashtekar Hamiltonian [8] in its original complex version, with the self-dual part of the Lorentz connection as the fundamental variable.

\footnotetext{
${ }^{3}$ This is a condition that does not change the dimensionality of the configuration space, by all means similar to requiring the invertibility of the metric in Einstein-Cartan.
} 
Interestingly, the structure of the constraint algebra, and with it the number of physical degrees of freedom, is unchanged if one replaces the constant $\lambda$ by a functional $\lambda\left(\varphi^{i j}\right)$ where $\varphi^{i j}$ is the symmetric, trace-free spacetime scalar built from $\epsilon_{k l}^{i} F_{a b}^{j} \Pi^{a k} \Pi^{b l}$. This was noticed in [19, 72], and has recently been pointed out again in $[57,59]$. We will come back to it below.

Quantization. The canonical quantization of this theory is the original program of loop quantum gravity in complex variables [8,91], where one looks for an Hilbert space of functionals of the Ashtekar self-dual connection. Notoriously, the main difficulty with this approach lies in the reality constraints. The reality conditions [28] are quadratic in the fields, and pose no particular problem at the classical level. However, they have so far provided a stumbling block for the quantum theory, and the program has then switched towards the use of a real connection, which is related to the non-chiral Plebanski action to be discussed below. For the Euclidean case, where reality conditions are not needed, a simplicial path integral was introduced in [79].

Second order BF action. One remarkable fact about $\mathrm{SU}(2) \mathrm{BF}$ theory is that it is always possible to explicitly and uniquely solve the compatibility condition $d_{\omega} B=0$, as long as $B$ is assumed to be non degenerate, even if the simplicity constraint is not satisfied. There exist a unique compatible connection $\omega(B)[20,38,52,58]$, given by

$$
\omega_{\mu}^{i}(B)=\frac{1}{4 e} \epsilon^{\rho \sigma \lambda \tau} B_{\lambda \tau}^{i} B_{j \rho \mu} \nabla^{\nu} B_{\nu \sigma}^{j} .
$$

Here the indices are raised and lowered with the Urbantke metric, $e$ is the determinant of its tetrad and $\nabla$ its covariant derivative. Using this result, one can give a second order formulation of the $\mathrm{BF}$ action (1). This formulation becomes particularly interesting if one uses the parametrization (6) for $B$ in terms of the Urbantke metric and the scalar fields $b_{a}^{i}$ or their metric $q_{a b}$. This was first proposed in [45] and in that case, the curvature of (9) includes the Riemann tensor of the metric. Therefore, one can obtain a second order formulation of $\mathrm{SU}(2)$ $\mathrm{BF}$ theory as an action for a metric and scalar fields, without any constraint needed. The result can be conveniently written using the internal unimodular metric $q_{a b}$ as follows [45],

$$
S\left(e_{\mu}^{I}, q_{a b}\right)=\frac{\eta \epsilon}{4} \int e R_{\epsilon}^{a b}(e)\left(\hat{q} \delta_{a b}-\hat{q}_{a b}\right)+\frac{1}{2} e \nabla_{\epsilon}^{\mu} q_{a b} C_{\epsilon \mu \nu}^{a b c d} \nabla_{\epsilon}^{\nu} q_{c d}-\epsilon \lambda \int e q .
$$

Here $\hat{q}_{a b}$ is the inverse of $q_{a b}$, with trace $\hat{q}$ (internal indices are still raised and lowered with the identity metric),

$$
R_{\epsilon}^{a b}(e)=\frac{1}{2} \Sigma_{\epsilon \mu \nu}^{a}(e) \Sigma_{\epsilon \rho \sigma}^{b}(e) R_{\mu \nu \rho \sigma}(e)
$$

is the self- or antiself-dual (resp. for $\epsilon= \pm$ ) part of the Riemann tensor, $\nabla_{\epsilon \mu}$ is the covariant derivative with respect to the spin connection $\gamma_{\epsilon b}^{a}(e)=\epsilon_{b c}^{a} P_{\epsilon I J}^{c} \omega^{I J}(e)$, and finally the kernel of the kinetic term is given by

$$
C_{\epsilon \mu \nu}^{a b c d}\left(e_{\mu}^{I}, q_{a b}\right) \equiv\left(\delta^{a d} \delta^{b c}-\frac{1}{2} \delta^{a b} \delta^{c d}\right) g_{\mu \nu}+\left(\delta^{b c} \epsilon_{g}^{a d}-\hat{q}^{b c} \epsilon^{a d f} q_{f g}\right) \Sigma_{\epsilon \mu \nu}^{g}(e) .
$$

The form (10) shows that BF theory, which is topological, can be formulated in terms of the Riemann tensor (11) of a space-time metric, coupled to scalar fields, that form the component of a internal SL(3) metric, which posses their own dynamics. The presence of these extra terms is crucial to have the shift symmetry (2), which is what eliminates any local degrees of freedom present a priori in the metric and the $b$ scalars. The metricity constraint (7) then plays a double role: on the one hand, it freezes the scalars. On the other hand, it is responsible for breaking the shift symmetry, leaving the local gauge and diffeomorphisms. Imposing the metricity constraint $q_{a b}=\delta_{a b}$, it is easily verified that (10) reduces to the Einstein-Hilbert Lagrangian $\eta \epsilon / 2 e[R(e)-6 \eta \lambda]$. 
This construction also gives a geometric interpretation to the scalars $b$. In fact, one can use the triad $b$ to introduce a new connection acting on the internal indices, as

$$
A^{a}{ }_{b} \equiv \hat{b}_{j}^{a} d_{\omega} b_{b}^{j}
$$

It is then easy to show that the new connection satisfies

$$
d_{A} \Sigma_{\epsilon}^{a}(e)=0, \quad d_{A} q_{a b}=0
$$

That is, $A$ is an $\mathrm{SL}(3)$ connection, torsion-free, but non-metric with respect to the local structure group $\mathrm{SU}(2)$. In this sense, the $b$ describe the non-metricity of the torsion-free connection $A$.

Modified theories of gravity. The discussion above pointed out the importance of breaking the shift symmetry in order to get propagation of local degrees of freedom. A generic way of doing so is to add a potential term for $\phi$ to (8), or equivalently, a potential for $B$ to the BF action,

$$
S(B, \omega)=\int B_{i} \wedge F^{i}(\omega)+\left(V(B)-\frac{\lambda}{2}\right) B_{i} \wedge B^{i}
$$

The potential $V(B)$ breaks the shift symmetry, leaving diffeomorphisms and local gauge transformations as symmetries of the action. Notice that it has to be a functional of (scalar contractions of) $B^{i} \wedge B^{j}$, so effectively $V(B)=V\left(q, q_{a b}^{2}\right)$. This class of actions has been introduced by Krasnov [59], and is related to the earlier formulations [19, 27, 72]. In the light of (10), we see that the result is an action for a metric and additional scalar field, with dynamics different from general relativity and driven by the precise form of $V(B)$.

An appealing aspect of (12) is that both BF and Plebanski's actions are special cases thereof, obtained respectively for a vanishing potential, and for a singular potential imposing (7) as a constraint. As emphasized e.g. in [58], this fact provides a well-defined scheme where BF theory could arise as the ultra-violet fixed point of low-energy general relativity, a possibility entertained in the literature (e.g. [82]). But what is really remarkable, is that for any potential, the theory still describes only two degrees of freedom. This can be shown through a canonical analysis $[57,59]$, which gives exactly what was anticipated in a previous subsection. The result makes (12) unlike other modifications of general relativity: In actions with a metric tensor as the unique field, modifications require the introduction of higher order diffeomorphism invariants, which in turn carry higher order derivatives, leading in general to extra degrees of freedom $[37,88]$. What allows the absence of extra degrees of freedom in this context is the presence of an infinite number of higher derivatives. Indeed, as shown first in [45] and furtherly developed in [58], the $b$ scalars can be integrated out, order by order in perturbation theory, thus obtaining a theory of the metric alone and with only two degrees of freedom, but at the price of generating an infinite number of higher derivatives terms. Some possible applications of this class of actions, both as modified classical theories of gravity, as grand unified theories, and as quantum mechanical effective theories, are discussed in the literature [56, 58, 60, 61].

\section{Non-chiral Plebanski action}

The Plebanski action can be formulated also using the full Lorentz group $\operatorname{SO}(3, \sigma)[28,36,80]$. In this non-chiral, or covariant, version the fundamental fields are $\left(B^{I J}, \omega^{I J}\right), I=0, \ldots, 3$. The key expression (4) for the Urbantke metric can be straightforwardly generalized to this case, but now two possible metrics exist. This is simply a consequence of the fact that the tensor product of three adjoint representations of the algebra admits two singlets. A basis in this two dimensional vector space is provided by the tensors $\eta_{N[I} \eta_{J] M K L}$ and $\eta_{N[I} \epsilon_{J] M K L}$, where $\epsilon_{I J K L}$ is 
the completely antisymmetric tensor and we defined the identity $\eta_{I J K L}=\frac{1}{2}\left(\eta_{I K} \eta_{J L}-\eta_{I L} \eta_{J K}\right)$. Accordingly, we have a right-handed Urbantke metric $g_{\mu \nu}^{(+)}$and a left-handed $g_{\mu \nu}^{(-)}$,

$$
\sqrt{g^{( \pm)}} g_{\mu \nu}^{( \pm)}=\frac{1}{12} \eta_{I N}\left(\eta_{J M K L} \pm \frac{\sqrt{\sigma}}{2} \epsilon_{J M K L}\right) \epsilon^{\alpha \beta \gamma \delta} B_{\mu \alpha}^{I J} B_{\beta \gamma}^{K L} B_{\delta \nu}^{M N}
$$

and $\sigma= \pm 1$ is the spacetime signature.

$B^{I J}$ can be immediately parametrized in terms of these two Urbantke metrics, using the left/right decomposition and applying twice (6). As the right- and left-handed parts are independent, the two sectors have independent triads and tetrads, say $b_{a}^{i}, \bar{b}_{a}^{i}$ and $e_{\mu}^{I}$, $\bar{e}_{\mu}^{I}$. Correspondingly, we have

$$
B^{I J}=P_{(+)}^{I J} b_{a}^{i} \Sigma_{(+)}^{a}(e)+\eta P_{(-)}^{I J} \bar{b}_{a}^{i} \Sigma_{(-)}^{a}(\bar{e}),
$$

where the Plebanski 2-forms encode the two metrics $g_{\mu \nu}^{(+)}=e_{\mu}^{I} e_{\nu}^{J} \delta_{I J}$ and $g_{\mu \nu}^{(+)}=\eta \bar{g}_{\mu \nu}=\eta \bar{e}_{\mu}^{I} \bar{e}_{\nu}^{J} \delta_{I J}$, and we dropped an irrelevant overall sign.

In the previous $\mathrm{SU}(2)$ case, the reduction of the 18 components of $B$ to a metric up to an $\mathrm{SU}(2)$ rotation required 5 constraints, see (7). We now have 36 initial components, and 6 internal gauge freedoms. We thus need 20 constraints: 10 to freeze the $b$ and $\bar{b}$ scalars, and 10 to identify $g$ and $\bar{g}$ into a unique metric. This can be immediately achieved with a covariant version of $(8)[28,36,80,81]$

$$
S(B, \omega)=\int B_{I J} \wedge F^{I J}+\frac{\Lambda}{4} \epsilon_{K L}^{I J} B_{I J} \wedge B^{K L}+\frac{1}{2} \phi_{I J K L} B^{I J} \wedge B^{K L} .
$$

The Lagrange multiplier $\phi_{I J K L}$ is by definition symmetric under the exchange of the first and second pair of indices. Since it is also antisymmetric within each pair, this leaves 21 independent components. This is one too many, thus one needs to remove one component if we want twenty constraints. The standard choice in the literature is to take the additional condition $\epsilon^{I J K L} \phi_{I J K L}=0$. The multiplier then decomposes into irreducible representations as $\phi \in(\mathbf{2}, \mathbf{0}) \oplus(\mathbf{0}, \mathbf{2}) \oplus(\mathbf{1}, \mathbf{1}) \oplus(\mathbf{0}, \mathbf{0})$, giving rise to corresponding constraints, known as simplicity constraints. For our purposes, it is sufficient to give an overview of their role, without looking at their explicit form. The five-dimensional self-dual representation $(\mathbf{2}, \mathbf{0})$ is again the constraint $(7)$ for the purely self-dual part of $B^{I J}$, thus it freezes the $b$ scalars in (14). Similarly, the $(\mathbf{0 , 2})$ freezes the $\bar{b}$. The ten-dimensional constraints in the representations $(\mathbf{1}, \mathbf{1}) \oplus(\mathbf{0}, \mathbf{0})$ impose the coincidence of the tetrads, $e_{\mu}^{I} \equiv \bar{e}_{\mu}^{I}$, by requiring the orthogonality of $\Sigma_{(+)}(e)$ and $\Sigma_{(-)}(\bar{e})$. See [87] for details. Finally, the sign $\eta$ remains free. Hence, the Urbantke metrics coincide up to a sign, $g_{\mu \nu}^{(+)}=\eta g_{\mu \nu}^{(-)}$. Plugging this result in (14), the two sectors $\eta= \pm 1$ give respectively

$$
B^{I J}=e^{I} \wedge e^{J}, \quad B^{I J}=\frac{1}{2} \epsilon_{K L}^{I J} e^{K} \wedge e^{L} .
$$

The theories described by these solutions can be examined simply by looking at the reduced actions for $e$ and $\omega$, which read respectively

$$
\begin{aligned}
& S_{\mathrm{top}}\left(e_{\mu}^{I}, \omega_{\mu}^{I J}\right)=\int \operatorname{tr}[e \wedge e \wedge F(\omega)]+6 \Lambda e, \\
& S_{\mathrm{EC}}\left(e_{\mu}^{I}, \omega_{\mu}^{I J}\right)=\int \operatorname{tr}[\star e \wedge e \wedge F(\omega)]+6 \Lambda e,
\end{aligned}
$$

where $\star=1 / 2 \epsilon_{K L}^{I J}$. The first action describes a topological theory, with no local degrees of freedom, see e.g. [64]. The second is the Einstein-Cartan action for general relativity. This is how the non-chiral Plebanski action describes gravity. See also [86] for the equivalence at the level of field equations. 
With respect to the self-dual action, the non-chiral formulation has the advantage that no additional reality conditions are needed in the Lorentzian case. In fact, although the individual Urbantke metrics (13) are complex when $B$ is real (an $i$ appears in front of the epsilon tensor), the final tetrad (16) is automatically real once the simplicity constraints are imposed. The other important aspect of this formulation is to be related to loop quantum gravity in real variables, as we now review.

Including the Immirzi parameter. The Lagrangians appearing in (17) can be considered in a unique action principle,

$$
S=\int \operatorname{tr}\left[P_{\gamma} e \wedge e \wedge F(\omega)\right]+6 \Lambda e, \quad P_{\gamma}=\frac{1}{\gamma}+\star,
$$

where the coupling constant $\gamma$ can be identified with the Immirzi parameter of loop quantum gravity, up to a topological term [35]. The Plebanski action (15) can be easily adapted to reduce to (18). The simplest way to do so is to include the second invariant in the kinetic term of (15), that is make the replacement

$$
\operatorname{tr}[B \wedge F] \rightarrow \operatorname{tr}\left[P_{\gamma} B \wedge F\right]
$$

Now both solutions (16) give an action like (18). Alternatively, one can choose the missing component of the Lagrange multiplier to be a linear combination of the two scalars $\delta^{I J K L} \phi_{I J K L}$ and $\epsilon^{I J K L} \phi_{I J K L}$. Then (16) is replaced by the unique solution $B=P_{\gamma} e \wedge e$, and again an action like (18) is recovered. See [29, 86] for discussions of these alternative cases. For more on the non-chiral Plebanski action, see e.g. [11, 70, 71, 74, 81, 95].

Canonical analysis. The canonical analysis of this theory has again second class constraints, but the analysis is considerably more involved than for the self-dual case. We do not present the details here, rather refer the reader to [5, 26, 95]. Again, the solution of the second class constraints, with or without time gauge, is given by an $\mathrm{SU}(2)$ connection, the Ashtekar-Barbero connection $[12,55]$. See $[6,14,32,51,54]$. This connection depends on the Immirzi parameter, and should be viewed as an auxiliary field, not straightforwardly related to the initial Lorentz group. The resulting theory has the phase space of a non-abelian gauge theory, and marks the starting point of loop quantum gravity $[9,39,83,91]^{4}$. It is interesting to add that canonically, the simplicity constraints can also be given a linear version. This linear formulation is at the root of the new spin foam models [43, 44, 46, 65].

Recently in $[23,24]$, it has also been proposed to approach the second class constraints via a different procedure, known as "gauge unfixing" [7, 53, 69]. That is, one views the second class constraints as the gauge-fixing part of a larger system with only first class constraints. This procedure typically involves a more complicated Hamiltonian, however the results of [23, 24] indicate that interesting new insights can be achieved this way, including a generalization of the loop quantum gravity techniques to higher dimensions and supergravity.

Quantization. The canonical quantization of the action (15), in a non-perturbative and background-independent way, is the program of loop quantum gravity in real variables. The same action is also the typical starting point for spin foam models [75]. The perturbative quantization of (18) has been studied in [17, 18], bringing to light the renormalization of the Immirzi parameter. Possible roles of the Immirzi parameter have been considered in the coupling to fermions [2, 49, 67, 76], and in cosmology [21].

Relaxed constraints and bi-metric theories of gravity. The above discussion highlights the fact that the non-chiral Plebanski action is naturally a theory of two metrics. It is only the presence of the simplicity constraints that forces the two metrics to coincide. One can consider

\footnotetext{
${ }^{4}$ An alternative solution has been proposed in [1,3]. See [4] for a recent overview.
} 
a modification of the theory along the lines of (12), where the constraints are replaced by a potential for $B$,

$$
S(B, \omega)=\int B_{I J} \wedge F^{I J}+\left(V(B)+\frac{\Lambda}{4}\right) \epsilon_{K L}^{I J} B_{I J} \wedge B^{K L} .
$$

In the absence of constraints, the two Urbantke metrics are independent and dynamical. In this case, extra degrees of freedom can be expected. This was indeed shown in [5], where a canonical analysis showed that for generic potentials, one has eight degrees of freedom. This was further clarified in [87], where using (14) and (10), it was shown that the modified Plebanski theory is equivalent to a bi-metric theory of gravity, plus the additional $b$ and $\bar{b}$ scalars. The interpretation of the eight physical degrees of freedom turned out to be a massless graviton, a massive spin-2 particle, and a scalar ${ }^{5}$. If (19) is extended to include the Immirzi parameter, the mass turns out to depend on it [16].

Interestingly, this is the same spectrum of pure bi-metric theories [34]. That is, one seems to be able to put all the relevant degrees of freedom in the metrics, without the scalars, likewise to the self-dual theory (see discussion in the previous section). On the other hand, the structure of a generic potential, to be built out of scalar invariants of $Q^{I J K L}=B^{I J} \wedge B^{K L}$, is now much richer. In particular, classes of potentials with lesser than eight degrees of freedom can be identified. These includes for instance Pauli-Fierz mass terms, and simpler scalar-tensor theories. These aspects are investigated in $[15,16]$, and we refer the reader to these references for further details, as well as discussions on the different reality conditions possible in the modified context.

Concerning applications, actions of this type can arise as effective descriptions of spin foam models of quantum gravity (e.g. [41]), and have been also used in grand unified theories, see $[63,85]$, where it resonates with similar ideas reviewed in [73]. More in general, bi-metric theories of gravity are interesting in the context of modified theories of gravity [33].

\section{MacDowell-Mansouri action}

A different mechanism is to introduce a tetrad through some components of the connection, as it happens in the Chern-Simons formulation of $2+1$ gravity [98]. The specific choice of gauge group depends on the spacetime signature, and on the sign of the cosmological constant one is interested in. In the case of a 4 d Lorentzian spacetime, one takes as $G$ the De Sitter group $\mathrm{SO}(4,1)$ for $\Lambda>0$, and the anti-de Sitter group $\mathrm{SO}(3,2)$ for $\Lambda<0$. For Euclidean signature, the respective choices are $\mathrm{SO}(5)$ and $\mathrm{SO}(4,1)$. To be specific, in the following we will consider Euclidean signature and positive $\Lambda$, but the results are easily generalized. Seen as a vector space, the Lie algebra splits as follows,

$$
\mathfrak{s o}(5)=\mathfrak{s o}(4) \oplus \mathbb{R}^{4},
$$

where the homogeneous space $\mathbb{R}^{4} \simeq \mathrm{SO}(5) / \mathrm{SO}(4)$ is spanned by the generators of translations. The splitting is orthogonal with respect to the Killing form, and $\mathfrak{s o}(4)$-invariant. It lets us introduce a tetrad as the vectorial part of the larger connection. To see it, let $A^{\alpha \beta}$ be the initial $\mathfrak{g}$ connection, $\alpha=0, \ldots, 4$, and let $I=0, \ldots, 3$. We then introduce the components

$$
A^{I J}=\omega^{I J}, \quad A^{I 4}=\frac{1}{\ell} e^{I} .
$$

\footnotetext{
${ }^{5}$ This identification is made via a perturbative expansion around the "bi-flat" background, $g_{\mu \nu}=\eta_{\mu \nu}+h_{\mu \nu}$, $\bar{g}_{\mu \nu}=\eta_{\mu \nu}+\bar{h}_{\mu \nu}$. Then one can consider the following linear combinations, $h_{\mu \nu}^{ \pm}=h_{\mu \nu} \pm \bar{h}_{\mu \nu}$. Since $h_{\mu \nu}^{-}$is invariant under diffeomorphisms, its masslessness is no longer protected by the symmetries. A generic potential term in (19) will result in a non-zero mass. Such a field in general propagates 6 degrees of freedom, corresponding to a spin-2 and a spin-0 particle. See $[16,87]$ for more details.
} 
Here $\ell$ is a constant with dimensions of a length, necessary to give both the tetrad and the connection canonical dimensions. It will turn out to be related to the cosmological constant via $\ell^{2}=3 / \Lambda .{ }^{6}$ That is, we view the $\mathrm{SO}(4)$ connection and the tetrad as two pieces of a unique $\mathfrak{g}$ connection, as one does for Chern-Simons gravity in $2+1$. A simple calculation gives

$$
F^{I J}(A)=F^{I J}(\omega)-\frac{1}{\ell^{2}} e^{I} \wedge e^{J}, \quad F^{I 4}=\frac{1}{\ell} d_{\omega} e^{I} .
$$

The equation $F^{\alpha \beta}(A)=0$ of BF theory alone thus implies de Sitter spacetime as the unique non-degenerate metric solution. To find all the solutions of general relativity, one needs to break the symmetry down to $\mathrm{SO}(4)$. This can be done adding an extra term in the BF action, which introduces a direction in the internal space:

$$
S=\int B_{\alpha \beta} \wedge F^{\alpha \beta}-\frac{\lambda}{2} B_{\alpha \beta} \wedge B^{\alpha \beta}-\frac{1}{2} \epsilon_{\alpha \beta \gamma \delta \varepsilon} v^{\varepsilon} B^{\alpha \beta} \wedge B^{\gamma \delta} .
$$

The extra term breaks not only $\mathrm{SO}(5)$, but also the shift symmetry (2), thus local degrees of freedom can be expected. Notice that with respect to the previous Plebanski formulation, we are adding here a potential-like term, and not a constraint. Here $v^{\alpha}=(0,0,0,0, v)$, consistently with the identifications (20).

Since the action is quadratic in $B$, the field can be trivially eliminated using its field equations, without any conditions on the remaining fields $\omega$ and $e$. Substituting back into the action, one obtains

$$
S_{M M}(A)=\int \frac{v}{2\left(v^{2}-\lambda^{2}\right)} \operatorname{tr}[\star F \wedge F]-\frac{\lambda}{2\left(v^{2}-\lambda^{2}\right)} \operatorname{tr}[F \wedge F]+\frac{1}{\lambda} F_{I 4} \wedge F^{I 4},
$$

where in the first two terms $F$ is the $\mathrm{SO}(4)$ curvature, and the $\mathrm{SO}(4)$ Hodge start $\star$ is embedded in $\mathrm{SO}(5)$ via $\epsilon_{I J K L 4}=\epsilon_{I J K L}$. The first term is the MacDowell-Mansouri action for gravity, introduced in [66] independently of the connection with BF theory. The remaining two are topological terms that do not affect the field equations. Hence, (22) provides a BF-like formulation of the MacDowell-Mansouri action. The equivalence with general relativity is then established thanks to the identities (21). After some simple algebra, one arrives at the form

$$
S=\int \frac{1}{G} \operatorname{tr}\left[P_{\gamma} e \wedge e \wedge F(\omega)\right]-\frac{\Lambda}{6 G} e+c_{1} E(\omega)+c_{2} P(\omega)+c_{3} N Y(e, \omega),
$$

where the last three terms are the topological invariants Euler, Pontryagin and Nieh-Yan, and the coefficients are given explicitly by

$$
\gamma=\frac{\lambda}{v}, \quad G \Lambda=\frac{3\left(v^{2}-\lambda^{2}\right)}{v}, \quad c_{1}=\frac{v}{4\left(v^{2}-\lambda^{2}\right)}, \quad c_{2}=-\frac{\lambda}{2\left(v^{2}-\lambda^{2}\right)}, \quad c_{3}=\frac{1}{\lambda},
$$

where we identified $\Lambda=3 / \ell^{2}$. Up to topological terms ${ }^{7}$, we obtained the Einstein-Cartan action for general relativity. We remark that the action principle has no scale to begin with, only the dimensionless quantities $\lambda$ and $v$. A scale, $\ell$, is introduced when splitting the components of $A$ into connection and tetrad, and it sets the cosmological constant scale.

The procedure of splitting the components of the connection into a smaller connection and a tetrad is reminiscent of the Chern-Simons formulation of three-dimensional gravity. However, unlike Chern-Simons, the relevant action principle is not invariant under the initial Lie algebra:

\footnotetext{
${ }^{6}$ An alternative formulation considers the possibility of interpreting $\ell$ as the Planck length [92], but we will not discuss this possibility here.

${ }^{7}$ It can also be observed that the six invariants in (24) are the only ones that can be written without requiring invertibility of the tetrad, or auxiliary fields.
} 
The symmetry is explicitly broken by the selection of a direction in the internal space. Efforts to try to make this dynamical have been discussed e.g. in [89, 93] and [96]. The typical difficulty is that the larger theory in which $\mathrm{SO}(5)$ is spontaneously broken possesses extra degrees of freedom, and its stability and unitarity have not been studied. An alternative viewpoint has been put forward in [50], where it was argued that the BF formulation (22) exhibits a spontaneous symmetry breaking mechanism that comes from integrating in the path integral over the $\mathrm{SO}(5)$ gauge degrees of freedom.

Many aspects of this formulation of gravity have been studied in the recent literature. These include symmetry properties [42], a geometric interpretation of the de Sitter local gauge group [97], the study of generalized solutions [78], as well as grand unified theories [62]. Finally, the mechanism can be extended to the SL(5) group [68].

Quantization. The action (23) looks promising for quantization, given its Yang-Mills form and dimensionless coupling constant. However, the situation is not so simple. In fact, in the naive form (23) in which the symmetry is explicitly broken, the action is de facto equivalent to (24) and quantization stumbles upon the usual difficulties. On the other hand, it was suggested in [50] to take (22) as the starting point, and consider a quantum path integral defined as a "topological" expansion around the BF kinetic term. This idea is quite intriguing, but it is challenged by difficulties with the gauge-fixing procedure: the zeroth order of the expansion has a different symmetry than the successive orders. See also [84] and [31] on this.

\section{Outlook}

One of the key difficulties with general relativity is the high non-linearity of its field equations. This complexity is enhanced further in the Einstein-Hilbert action principle, which is non-polynomial in the fundamental field, the metric. To obtain a polynomial action, one has to expand the metric around a fixed background. Then the perturbations can be quantized, but the theory is not renormalizable. An important line of research in quantum gravity imputes this failure to the background-dependent, perturbative methods, and seeks a backgroundindependent formulation. When seeking for alternative approaches, the use of different fundamental variables with simpler actions is a useful guiding principle. In this respect, the relation of general relativity with BF theory appears very promising. The work appeared so far in the literature has unraveled the deepest level of such a classical relation, and introduced new tools and ideas to push forward the investigation of gravity in these variables. These results can be of benefit to approaches such as loop quantum gravity and spin foam models.

\section{References}

[1] Alexandrov S., Choice of connection in loop quantum gravity, Phys. Rev. D 65 (2002), 024011, 7 pages, gr-qc/0107071.

[2] Alexandrov S., The Immirzi parameter and fermions with non-minimal coupling, Classical Quantum Gravity 25 (2008), 145012, 4 pages, arXiv:0802.1221.

[3] Alexandrov S., Buffenoir E., Roche P., Plebanski theory and covariant canonical formulation, Classical Quantum Gravity 24 (2007), 2809-2824, gr-qc/0612071.

[4] Alexandrov S., Geiller M., Noui K., Spin foams and canonical quantization, arXiv:1112.1961.

[5] Alexandrov S., Krasnov K., Hamiltonian analysis of non-chiral Plebanski theory and its generalizations, Classical Quantum Gravity 26 (2009), 055005, 10 pages, arXiv:0809.4763.

[6] Alexandrov S., Livine E.R., SU(2) loop quantum gravity seen from covariant theory, Phys. Rev. D 67 (2003), 044009, 15 pages, gr-qc/0209105.

[7] Anishetty R., Vytheeswaran A.S., Gauge invariance in second-class constrained systems, J. Phys. A: Math. Gen. 26 (1993), 5613-5619. 
[8] Ashtekar A., New Hamiltonian formulation of general relativity, Phys. Rev. D 36 (1987), 1587-1602.

[9] Ashtekar A., Lewandowski J., Background independent quantum gravity: a status report, Classical Quantum Gravity 21 (2004), R53-R152, gr-qc/0404018.

[10] Baez J.C., An introduction to spin foam models of BF theory and quantum gravity, in Geometry and Quantum Physics (Schladming, 1999), Lecture Notes in Phys., Vol. 543, Editors H. Gausterer, H. Grosse, Springer, Berlin, 2000, 25-93, gr-qc/9905087.

[11] Baratin A., Oriti D., Group field theory and simplicial gravity path integrals: a model for Holst-Plebanski gravity, Phys. Rev. D 85 (2012), 044003, 15 pages, arXiv:1111.5842.

[12] Barbero G. J.F., Real Ashtekar variables for Lorentzian signature space-times, Phys. Rev. D 51 (1995), 5507-5510, gr-qc/9410014.

[13] Barrett J.W., Naish-Guzman I., The Ponzano-Regge model, Classical Quantum Gravity 26 (2009), 155014, 48 pages, arXiv:0803.3319.

[14] Barros e Sá N., Hamiltonian analysis of general relativity with the Immirzi parameter, Internat. J. Modern Phys. D 10 (2001), 261-272, gr-qc/0006013.

[15] Beke D., Scalar-tensor theories from $\Lambda(\phi)$ Plebanski gravity, arXiv:1111.1139.

[16] Beke D., Palmisano G., Speziale S., Pauli-Fierz mass term in modified Plebanski gravity, J. High Energy Phys. 2012 (2012), no. 3, 069, 28 pages, arXiv:1112.4051.

[17] Benedetti D., Speziale S., Perturbative quantum gravity with the Immirzi parameter, J. High Energy Phys. 2011 (2011), no. 6, 107, 31 pages, arXiv:1104.4028.

[18] Benedetti D., Speziale S., Perturbative running of the Immirzi parameter, arXiv:1111.0884.

[19] Bengtsson I., The cosmological constants, Phys. Lett. B 254 (1991), 55-60.

[20] Bengtsson I., 2-form geometry and the 't Hooft-Plebanski action, Classical Quantum Gravity 12 (1995), 1581-1590, gr-qc/9502010.

[21] Bethke L., Magueijo J., Chirality of tensor perturbations for complex values of the Immirzi parameter, arXiv:1108.0816.

[22] Birmingham D., Blau M., Rakowski M., Thompson G., Topological field theory, Phys. Rep. 209 (1991), 129-340.

[23] Bodendorfer N., Thiemann T., Thurn A., New variables for classical and quantum gravity in all dimensions. I. Hamiltonian analysis, arXiv:1105.3703.

[24] Bodendorfer N., Thiemann T., Thurn A., On the implementation of the canonical quantum simplicity constraint, arXiv:1105.3708.

[25] Bonzom V., Smerlak M., Bubble divergences: sorting out topology from cell structure, Ann. Henri Poincaré 13 (2012), 185-208, arXiv:1103.3961.

[26] Buffenoir E., Henneaux M., Noui K., Roche P., Hamiltonian analysis of Plebanski theory, Classical Quantum Gravity 21 (2004), 5203-5220, gr-qc/0404041.

[27] Capovilla R., Generally covariant gauge theories, Nuclear Phys. B 373 (1992), 233-246.

[28] Capovilla R., Dell J., Jacobson T., Mason L., Self-dual 2-forms and gravity, Classical Quantum Gravity 8 (1991), 41-57.

[29] Capovilla R., Montesinos M., Prieto V.A., Rojas E., BF gravity and the Immirzi parameter, Classical Quantum Gravity 18 (2001), L49-L52, gr-qc/0102073.

[30] Cattaneo A.S., Cotta-Ramusino P., Fröhlich J., Martellini M., Topological BF theories in 3 and 4 dimensions, J. Math. Phys. 36 (1995), 6137-6160, hep-th/9505027.

[31] Cattaneo A.S., Cotta-Ramusino P., Fucito F., Martellini M., Rinaldi M., Tanzini A., Zeni M., Fourdimensional Yang-Mills theory as a deformation of topological BF theory, Comm. Math. Phys. 197 (1998), 571-621, hep-th/9705123.

[32] Cianfrani F., Montani G., Towards loop quantum gravity without the time gauge, Phys. Rev. Lett. 102 (2009), 091301, 4 pages, arXiv:0811.1916.

[33] Clifton T., Bañados M., Skordis C., The parameterized post-Newtonian limit of bimetric theories of gravity, Classical Quantum Gravity 27 (2010), 235020, 31 pages, arXiv:1006.5619.

[34] Damour T., Kogan I.I., Effective Lagrangians and universality classes of nonlinear bigravity, Phys. Rev. D 66 (2002), 104024, 17 pages, hep-th/0206042. 
[35] Date G., Kaul R.K., Sengupta S., Topological interpretation of Barbero-Immirzi parameter, Phys. Rev. D 79 (2009), 044008, 7 pages, arXiv:0811.4496.

[36] De Pietri R., Freidel L., so(4) Plebański action and relativistic spin-foam model, Classical Quantum Gravity 16 (1999), 2187-2196, gr-qc/9804071.

[37] Deruelle N., Sasaki M., Sendouda Y., Yamauchi D., Hamiltonian formulation of f(Riemann) theories of gravity, Progr. Theoret. Phys. 123 (2010), 169-185, arXiv:0908.0679.

[38] Deser S., Teitelboim C., Duality transformations of Abelian and non-Abelian gauge fields, Phys. Rev. D 13 (1976), 1592-1597.

[39] Dona P., Speziale S., Introductory lectures to loop quantum gravity, arXiv:1007.0402.

[40] Dunajski M., Solitons, instantons, and twistors, Oxford Graduate Texts in Mathematics, Vol. 19, Oxford University Press, Oxford, 2010.

[41] Dupuis M., Livine E.R., Holomorphic simplicity constraints for 4D spinfoam models, Classical Quantum Gravity 28 (2011), 215022, 32 pages, arXiv:1104.3683.

[42] Durka R., Kowalski-Glikman J., Gravity as a constrained BF theory: Noether charges and Immirzi parameter, Phys. Rev. D $\mathbf{8 3}$ (2011), 124011, 6 pages, arXiv:1103.2971.

[43] Engle J., Livine E., Pereira R., Rovelli C., LQG vertex with finite Immirzi parameter, Nuclear Phys. B 799 (2008), 136-149, arXiv:0711.0146.

[44] Engle J., Pereira R., Rovelli C., Loop-quantum-gravity vertex amplitude, Phys. Rev. Lett. 99 (2007), 161301, 4 pages, arXiv:0705.2388.

[45] Freidel L., Modified gravity without new degrees of freedom, arXiv:0812.3200.

[46] Freidel L., Krasnov K., A new spin foam model for 4D gravity, Classical Quantum Gravity 25 (2008), 125018, 36 pages, arXiv:0708.1595.

[47] Freidel L., Krasnov K., Puzio R., BF description of higher-dimensional gravity theories, Adv. Theor. Math. Phys. 3 (1999), 1289-1324, hep-th/9901069.

[48] Freidel L., Louapre D., Diffeomorphisms and spin foam models, Nuclear Phys. B 662 (2003), 279-298, gr-qc/0212001.

[49] Freidel L., Minic D., Takeuchi T., Quantum gravity, torsion, parity violation, and all that, Phys. Rev. D 72 (2005), 104002, 6 pages, hep-th/0507253.

[50] Freidel L., Starodubtsev A., Quantum gravity in terms of topological observables, hep-th/0501191.

[51] Geiller M., Lachieze-Rey M., Noui K., A new look at Lorentz-covariant loop quantum gravity, Phys. Rev. D 84 (2011), 044002, 19 pages, arXiv:1105.4194.

[52] Halpern M.B., Field-strength and dual variable formulations of gauge theory, Phys. Rev. D 19 (1979), 517-530.

[53] Henneaux M., Teitelboim C., Quantization of gauge systems, Princeton University Press, Princeton, NJ, 1992.

[54] Holst S., Barbero's Hamiltonian derived from a generalized Hilbert-Palatini action, Phys. Rev. D 53 (1996), 5966-5969, gr-qc/9511026.

[55] Immirzi G., Real and complex connections for canonical gravity, Classical Quantum Gravity 14 (1997), L177-L181, gr-qc/9612030.

[56] Ishibashi A., Speziale S., Spherically symmetric black holes in minimally modified self-dual gravity, Classical Quantum Gravity 26 (2009), 175005, 37 pages, arXiv:0904.3914.

[57] Krasnov K., Deformations of the constraint algebra of Ashtekar's Hamiltonian formulation of general relativity, Phys. Rev. Lett. 100 (2008), 081102, 4 pages, arXiv:0711.0090.

[58] Krasnov K., Effective metric Lagrangians from an underlying theory with two propagating degrees of freedom, Phys. Rev. D 81 (2010), 084026, 40 pages, arXiv:0911.4903.

[59] Krasnov K., Renormalizable non-metric quantum gravity?, hep-th/0611182.

[60] Krasnov K., Shtanov Y., Cosmological perturbations in a family of deformations of general relativity, J. Cosmol. Astropart. Phys. 2010 (2010), no. 6, 006, 42 pages, arXiv:1002.1210.

[61] Krasnov K., Shtanov Y., Halos of modified gravity, Internat. J. Modern Phys. D 17 (2008), 2555-2562, arXiv:0805.2668.

[62] Lisi A.G., An exceptionally simple theory of everything, arXiv:0711.0770. 
[63] Lisi A.G., Smolin L., Speziale S., Unification of gravity, gauge fields and Higgs bosons, J. Phys. A: Math. Theor. 43 (2010), 445401, 10 pages, arXiv:1004.4866.

[64] Liu L., Montesinos M., Perez A., Topological limit of gravity admitting an SU(2) connection formulation, Phys. Rev. D 81 (2010), 064033, 9 pages, arXiv:0906.4524.

[65] Livine E.R., Speziale S., Solving the simplicity constraints for spinfoam quantum gravity, Europhys. Lett. 81 (2008), 50004, 6 pages, arXiv:0708.1915.

[66] MacDowell S.W., Mansouri F., Unified geometric theory of gravity and supergravity, Phys. Rev. Lett. 38 (1977), 739-742.

[67] Mercuri S., Fermions in the Ashtekar-Barbero connection formalism for arbitrary values of the Immirzi parameter, Phys. Rev. D $\mathbf{7 3}$ (2006), 084016, 14 pages, gr-qc/0601013.

[68] Mielke E.W., Spontaneously broken topological SL(5, R) gauge theory with standard gravity emerging, Phys. Rev. D 83 (2011), 044004, 9 pages.

[69] Mitra P., Rajaraman R., Gauge-invariant reformulation of theories with second-class constraints, Ann. Physics 203 (1990), 157-172.

[70] Montesinos M., Alternative symplectic structures for $\mathrm{SO}(3,1)$ and $\mathrm{SO}(4)$ four-dimensional BF theories, Classical Quantum Gravity 23 (2006), 2267-2278, gr-qc/0603076.

[71] Montesinos M., Velázquez M., BF gravity with Immirzi parameter and cosmological constant, Phys. Rev. D 81 (2010), 044033, 4 pages, arXiv:1002.3836.

[72] Peldán P., Actions for gravity, with generalizations: a review, Classical Quantum Gravity 11 (1994), 10871132, gr-qc/9305011.

[73] Percacci R., Gravity from a particle physicists' perspective, PoS Proc. Sci. (2009), PoS(ISFTG2009), 011, 30 pages, arXiv:0910.5167.

[74] Perez A., Spin foam quantization of SO(4) Plebański's action, Adv. Theor. Math. Phys. 5 (2001), 947-968, gr-qc/0203058.

[75] Perez A., The spin foam approach to quantum gravity, Living Rev. Relativ., to appear, arXiv:1205.2019.

[76] Perez A., Rovelli C., Physical effects of the Immirzi parameter in loop quantum gravity, Phys. Rev. D 73 (2006), 044013, 3 pages, gr-qc/0505081.

[77] Plebanski J.F., On the separation of Einsteinian substructures, J. Math. Phys. 18 (1977), 2511-2520.

[78] Randono A., de Sitter spaces: topological ramifications of gravity as a gauge theory, Classical Quantum Gravity 27 (2010), 105008, 18 pages, arXiv:0909.5435.

[79] Reisenberger M.P., A left-handed simplicial action for Euclidean general relativity, Classical Quantum Gravity 14 (1997), 1753-1770, gr-qc/9609002.

[80] Reisenberger M.P., Classical Euclidean general relativity from "left-handed area = right-handed area", Classical Quantum Gravity 16 (1999), 1357-1371, gr-qc/9804061.

[81] Reisenberger M.P., New constraints for canonical general relativity, Nuclear Phys. B 457 (1995), 643-687, gr-qc/9505044.

[82] Rivasseau V., Towards renormalizing group field theory, PoS Proc. Sci. (2010), PoS(CNCFG2010), 004, 21 pages, arXiv:1103.1900.

[83] Rovelli C., Quantum gravity, Cambridge Monographs on Mathematical Physics, Cambridge University Press, Cambridge, 2004.

[84] Rovelli C., Speziale S., On the expansion of a quantum field theory around a topological sector, Gen. Relativity Gravitation 39 (2007), 167-178, gr-qc/0508106.

[85] Smolin L., Plebanski action extended to a unification of gravity and Yang-Mills theory, Phys. Rev. D 80 (2009), 124017, 6 pages, arXiv:0712.0977.

[86] Smolin L., Speziale S., Note on the Plebanski action with the cosmological constant and an Immirzi parameter, Phys. Rev. D $\mathbf{8 1}$ (2010), 024032, 6 pages, arXiv:0908.3388.

[87] Speziale S., Bimetric theory of gravity from the nonchiral Plebanski action, Phys. Rev. D 82 (2010), 064003, 17 pages, arXiv:1003.4701.

[88] Stelle K.S., Classical gravity with higher derivatives, Gen. Relativity Gravitation 9 (1978), 353-371.

[89] Stelle K.S., West P.C., de Sitter gauge invariance and the geometry of the Einstein-Cartan theory, J. Phys. A: Math. Gen. 12 (1979), L205-L210. 
[90] 't Hooft G., A chiral alternative to the vierbein field in general relativity, Nuclear Phys. B 357 (1991), 211-221.

[91] Thiemann T., Modern canonical quantum general relativity, Cambridge Monographs on Mathematical Physics, Cambridge University Press, Cambridge, 2007, gr-qc/0110034.

[92] Townsend P.K., Small-scale structure of spacetime as the origin of the gravitational constant, Phys. Rev. D 15 (1977), 2795-2801.

[93] Tseytlin A.A., Poincaré and de Sitter gauge theories of gravity with propagating torsion, Phys. Rev. D 26 (1982), 3327-3341.

[94] Urbantke H., On integrability properties of SU(2) Yang-Mills fields. I. Infinitesimal part, J. Math. Phys. 25 (1984), 2321-2324.

[95] Wieland W.M., Complex Ashtekar variables and reality conditions for Holst's action, Ann. Henri Poincaré 13 (2012), 425-448, arXiv:1012.1738.

[96] Wilczek F., Riemann-Einstein structure from volume and gauge symmetry, Phys. Rev. Lett. 80 (1998), 4851-4854, hep-th/9801184.

[97] Wise D.K., MacDowell-Mansouri gravity and Cartan geometry, Classical Quantum Gravity 27 (2010), 155010, 26 pages, gr-qc/0611154.

[98] Witten E., 2+1-dimensional gravity as an exactly soluble system, Nuclear Phys. B 311 (1988), 46-78.

[99] Zapata J.A., Topological lattice gravity using self-dual variables, Classical Quantum Gravity 13 (1996), 2617-2634, gr-qc/9603030. 